Bulletin of the Section of Logic

Volume 44:3/4 (2015), pp. 111-130

http://dx.doi.org/10.18778/0138-0680.44.3.4.02

Zofia Kostrzycka*

\title{
ON HALLDÉN COMPLETENESS OF MODAL LOGICS DETERMINED BY HOMOGENEOUS KRIPKE FRAMES
}

\begin{abstract}
Halldén complete modal logics are defined semantically. They have a nice characterization as they are determined by homogeneous Kripke frames.
\end{abstract}

\section{Introduction}

In this paper we continue research on modal logics which are Halldén complete. In paper [8] a class of Halldén incomplete logics among Brouwerian modal logics is described. The class has the cardinality of the continuum. In the current paper we present some positive results in this field. The method presented for showing Halldén completeness (and incompleteness) is applicable to other modal logics as well.

Definition 1. A logic $L$ is Halldén complete if

$$
\varphi \vee \psi \in L \text { implies } \varphi \in L \text { or } \psi \in L
$$

for all $\varphi$ and $\psi$ containing no common variables.

Halldén complete logics are often called Halldén reasonable. It is proven that the well known modal logics S4 and S5, KT and KTB, S4.3 are Halldén complete (see [12], [10], [1]). On the other side, it is known that the logics $\mathbf{S 1}-\mathbf{S 3}$ are Halldén incomplete [5].

*Supported by the NCN, research grant DEC-2013/09/B/HS1/00701 
Some other results concerning Halldén completeness of modal logics are the following:

- All normal extensions of S5 are Halldén complete [12].

- There is a continuum of Halldén complete logics in $N E X T(\mathbf{S 4})$ [3].

- There is a continuum of Halldén incomplete logics in $N E X T\left(\mathbf{K T B} \oplus \square^{2} p \rightarrow \square^{3} p\right)[8]$.

In this paper we give a semantic method of construction of Halldén complete modal logics. Let $\mathcal{K}$ be some class of Kripke frames. Formally, the logic determined by $\mathcal{K}$ is defined as follows:

$$
L(\mathcal{K}):=\{\alpha \in \text { Form }: \mathfrak{F} \models \alpha \text { for each } \mathfrak{F} \in \mathcal{K}\} .
$$

Note that the class $\mathcal{K}$ may consist of one Kripke frame only. Then we will write $L=L(\mathfrak{F})$. Hereafter, by 'frame' we mean a Kripke frame. As is well known, different classes of Kripke frames, determine different modal logics.

\begin{tabular}{|c|c|c|}
\hline & axioms & condition for $R$ in Kripke frames \\
\hline$K$ & $\square(p \rightarrow q) \rightarrow(\square p \rightarrow \square q)$ & no condition \\
$T$ & $\square p \rightarrow p$ & $R$ reflexive \\
$B$ & $p \rightarrow \square \triangleright p$ & $R$ symmetric \\
$4_{1}$ & $\square p \rightarrow \square \square p$ & $R$ transitive \\
\hline
\end{tabular}

The smallest normal modal logic is denoted by $\mathbf{K}$. For two normal modal $\operatorname{logics} L$ and $L^{\prime}, L^{\prime}$ is a normal extension of $L$ if $L \subset L^{\prime}$. The class of all normal extensions of $L$ is denoted by $N E X T(L)$. For a normal logic $L$ and a formula $\varphi$, the smallest normal extension of $L$ containing also $\varphi$ is denoted by $L \oplus \varphi$. Then one gets: $\mathbf{K T}:=\mathbf{K} \oplus T$, KTB $:=\mathbf{K T} \oplus B$, $\mathbf{S} 4:=\mathbf{K T} \oplus 4_{1}$ and $\mathbf{S} 5:=\mathbf{S} 4 \oplus B$.

In the paper we will also consider special kind of normal extension of the Brouwer logic KTB. We consider formulas

$$
\begin{gathered}
\left(4_{n}\right) \quad \square^{n} p \rightarrow \square^{n+1} p, \quad \text { for } n \geq 1 \\
\left.\left(\text { alt }_{n}\right):=\square p_{1} \vee \square\left(p_{1} \rightarrow p_{2}\right) \vee \ldots \vee \square\left(\left(p_{1} \wedge \ldots \wedge p_{n}\right) \rightarrow p_{n+1}\right)\right), n \geq 0,
\end{gathered}
$$

and logics KTB. $4_{\mathbf{n}}:=\mathbf{K T B} \oplus\left(4_{n}\right)$ and KTB.alt $\mathbf{n}:=\mathbf{K T B} \oplus\left(\right.$ alt $\left._{n}\right)$. Axioms $\left(4_{n}\right)$ are called $n$-transitivity axioms. Respectively in frames, the relation $R$ is $n$-transitive if

$$
\left(\operatorname{tran}_{n}\right) \quad \forall_{x, y}\left(\text { if } x R^{n+1} y \text { then } x R^{n} y\right)
$$


where the relation $R^{n}$ is the $n$-step accessibility relation defined below:

$$
\begin{array}{rll}
x R^{0} y & \text { iff } & x=y \\
x R^{n+1} y & \text { iff } & \exists_{z}\left(x R^{n} z \wedge z R y\right)
\end{array}
$$

Axioms $\left(\right.$ alt $\left._{n}\right)$ with $n \geq 1$ modally define the classes of frames in which each point sees at most $n$ points (possibly including itself).

Dealing with Kripke frames we need the notion of p-morphism.

Definition 2. Let $\mathfrak{F}_{1}:=\left\langle W_{1}, R_{1}\right\rangle$ and $\mathfrak{F}_{2}:=\left\langle W_{2}, R_{2}\right\rangle$ be Kripke frames. A map $f: W_{1} \rightarrow W_{2}$ is a p-morphism from $\mathfrak{F}_{1}$ to $\mathfrak{F}_{2}$, if it satisfies the following conditions:

(p1) $f$ maps $W_{1}$ onto $W_{2}$,

(p2) for all $x, y \in W_{1}, x R_{1} y$ implies $f(x) R_{2} f(y)$,

(p3) for each $x \in W_{1}$ and for each $a \in W_{2}$, if $f(x) R_{2} a$ then there exists $y \in W_{1}$ such that $x R_{1} y$ and $f(y)=a$.

It is said that the frame $\mathfrak{F}_{1}$ is reducible to $\mathfrak{F}_{2}$ or that the frame $\mathfrak{F}_{2}$ is a p-morphic reduct of $\mathfrak{F}_{1}$.

Then we may compare the strength of logics. The following lemma is a piece of logical folklore:

LEMMA 1. Let $\mathfrak{F}_{1}, \mathfrak{F}_{2}$ be Kripke frames. If there exists a p-morphism from $\mathfrak{F}_{1}$ to $\mathfrak{F}_{2}$ then $L\left(\mathfrak{F}_{1}\right) \subseteq L\left(\mathfrak{F}_{2}\right)$.

Proof. See [4], p. 31-32.

Below, we recall some algebraic notions connected with modal logics.

Definition 3. An algebra $\mathfrak{A}:=\langle A, \cap, \cup,-, I, 0,1\rangle$ is a modal algebra if $\langle A, \cap, \cup,-, 0,1\rangle$ is a Boolean algebra and the unary operator I satisfies the conditions:

(1) $I(1)=1$,

(2) $I(a \cap b)=I(a) \cap I(b)$ for any $a, b \in A$.

The best known class of modal algebras is the class of topological Boolean algebras. They characterize the logic $\mathbf{S} 4$ and its normal extensions. In the 
topological Boolean algebras ( $S 4$-algebras) the operator $I$ has to fulfill additionally the conditions

(3) $I(a) \leq a$

(4) $I(I(a))=I(a)$

for any $a \in A$ and behaves as the interior operator. The order $\leq$ is defined in the standard way: $a \leq b$ iff $a \cap b=a$.

Definition 4. A modal algebra $\mathfrak{A}:=\langle A, \cap, \cup,-, I, 0,1\rangle$ is called a KTBalgebra if the unary operator I satisfies the condition (3) for any $a \in A$ as well as:

(5) $a \leq I(-I(-a))$.

Similarly, if the operator $I$ satisfies the conditions (1), (2) and (3) then the algebra is called $K T$-algebra, whereas in the case of fulfilling (1), (2), (5) the appropriate algebra is $K B$-algebra.

Let $\mathfrak{A}:=\langle A, \cap, \cup,-, I, 0,1\rangle$ be a modal algebra. A non-empty set $\nabla \subseteq A$ is a filter in $\mathfrak{A}$ if the following conditions hold:

(i) If $a, b \in \nabla$ then $a \cap b \in \nabla$,

(ii) If $a \leq b$ and $a \in \nabla$ then $b \in \nabla$.

A filter $\nabla$ is an open filter in $\mathfrak{A}$ if additionally it holds:

(iii) If $a \in \nabla$ then $I(a) \in \nabla$.

Definition 5. A modal algebra is called simple if it does not contain any proper open filter.

For finite Kripke frames and finite modal algebras there is a nice duality between them. For a finite modal algebra $\mathfrak{A}$ we define the dual frame $\mathfrak{A}_{*}:=\left\langle W_{*}, R_{*}\right\rangle$ where $W_{*}$ is the set of atoms of algebra $\mathfrak{A}$ and $R_{*}$ is a binary relation defined for any $x, y \in W_{*}$ as follows:

$$
x R_{*} y \quad \text { iff } \forall_{z \in A}(x \leq I(z) \Rightarrow y \leq z) .
$$

It is known that both $\mathfrak{A}$ and $\mathfrak{A}_{*}$ validate the same formulas. Conversely, for each finite Kripke frame $\mathfrak{F}:=\langle W, R\rangle$ we define its dual algebra $\mathfrak{F}^{*}:=$ $\left\langle 2^{W}, \cap, \cup,-, I, \emptyset, W\right\rangle$ where for any $X \subseteq W$

$$
I(X)=\left\{x \in W: \forall_{y}(x R y \Rightarrow y \in X)\right\} .
$$


Similarly, both the frame $\mathfrak{F}$ and its dual algebra $\mathfrak{F}^{*}$ validate the same modal formulas. For more details see [4]. Moreover, for finite cases we have

$$
\left(\mathfrak{F}^{*}\right)_{*} \cong \mathfrak{F} \text { and }\left(\mathfrak{A}_{*}\right)^{*} \cong \mathfrak{A} .
$$

For infinite case there is only the isomorphism $\left(\mathfrak{A}_{*}\right)^{*} \cong \mathfrak{A}$.

For some special Kripke frames Lemma 1 may be strengthened to an equivalence.

LEMMA 2. Let $\mathfrak{F}_{1}, \mathfrak{F}_{2}$ be finite and connected Kripke frames such that their dual algebras are simple. Then $L\left(\mathfrak{F}_{1}\right) \subseteq L\left(\mathfrak{F}_{2}\right)$ iff there exists a p-morphism from $\mathfrak{F}_{1}$ to $\mathfrak{F}_{2}$.

Proof. It is proven by Jónsson's lemma, the congruence extension property of modal algebras, finiteness and simplicity of the dual algebra for $\mathfrak{F}_{1}$. For details, see for example [13] or [7].

Another tool for dealing with frames are characteristic formulas. They were first introduced for intuitionistic logic (and Heyting algebras) by Jankov [6], but later they were adopted to modal logics as well. Then, by the theory of duality, characteristic formulas are also used for Kripke frames. For each finite frame $\mathfrak{F}:=\langle W, R\rangle$ we define its diagram $\Delta_{\mathfrak{F}}$ as follows:

- for each element $a \in W$ we fix a distinct propositional variable $p_{a}$.

- $\Delta_{\mathfrak{F}}:=\left\{p_{a} \rightarrow \diamond p_{b}: a R b\right\} \cup\left\{p_{a} \rightarrow \neg \diamond p_{b}: \neg(a R b)\right\} \cup\left\{p_{a} \rightarrow \neg p_{b}:\right.$ $a \neq b\} \cup\left\{\bigvee_{x \in W} p_{x}\right\}$

The characteristic formula for the frame $\mathfrak{F}$ is defined $\delta_{\mathfrak{F}}:=\wedge \Delta_{\mathfrak{F}}$. If $\operatorname{card}(W)=n$ then we define for any $x \in W$ :

$$
\kappa_{x}:=\square^{n} \delta_{\mathfrak{F}} \wedge p_{x} .
$$

For finite frames with $\operatorname{card}(W)=n$ it holds:

the formula $\kappa_{x}$ is satisfiable at $x$.

\section{Logics determined by homogeneous frames}

Our key tool to recognize Halldén complete modal logics will be a theorem due to van Benthem and Humberstone from [1]. In the paper the authors consider many classes of frames in respect to determining Halldén complete 
logics. Among the investigated classes the class of homogeneous frames is also studied.

Definition 6. A Kripke frame $\mathfrak{F}:=\langle W, R\rangle$ is homogeneous if for any $x, y \in W$ there exists an automorphism $f$ of $\langle W, R\rangle$ with $f(x)=y$.

As a conclusion of more general theorem (Theorem 1 from [1]) the following lemma is written.

Lemma 3. If a modal logic $L$ is determined by one Kripke frame, which is homogeneous, then $L$ is Halldén complete.

Proof. It is a simplification of the proof of Theorem 1 from [1]. Let $L=L(\mathfrak{F})$ and $\mathfrak{F}:=\langle W, R\rangle$. Let $\varphi \vee \psi$ be some variable disjoint disjunction, such that $L \forall \varphi$ and $L \forall \psi$. Then there is a valuation $v_{1}$ on $\mathfrak{F}$ and a point $x \in W$ such that $(\mathfrak{F}, x) \not \forall_{v_{1}} \varphi$ and a valuation $v_{2}$ on $\mathfrak{F}$ and a point $y \in W$ such that $(\mathfrak{F}, y) \not \nvdash_{v_{2}} \psi$. But since the frame $\mathfrak{F}$ is homogeneous, then there is an automorphism $f$ such that $f(y)=x$. The new valuation for all variables $p_{i}$ of the formula $\psi$ are defined: $v^{*}\left(p_{i}, f(x)\right)=v_{2}\left(p_{i}, x\right)$ for all $x \in W$. There is no conflict with the valuation $v_{1}$ since $\varphi$ and $\psi$ have disjoint variables. We extend valuation $v^{*}$ on the set of variables $q_{i}$ of $\varphi$ : $v^{*}\left(q_{i}, x\right)=v_{2}\left(q_{i}, x\right)$. Then we get $(\mathfrak{F}, x) \not v_{v^{*}} \varphi \vee \psi$.

The above theorem may be strengthened to an equivalence for some special class of frames. We shall return to this problem in the next section.

Defining Halldén complete logics, we are however bounded by another theorem due to Lemmon [11]. For non-normal modal logics, the theorem is an equivalence. For normal extensions it has the form of implication only. Following Lemmon we say, that two logics $L_{1}, L_{2} \in N E X T(L)$ are incomparable, if $L_{1} \not \subset L_{2}$ and $L_{2} \not \subset L_{1}$.

TheOrem 1. Let $L_{1}, L_{2} \in N E X T(L)$ be two incomparable logics. Then the logic $L_{0}=L_{1} \cap L_{2}$ is Halldén incomplete.

Proof: We quote the proof from [11]. Let $L_{0}=L_{1} \cap L_{2}$ and there exist $\varphi$ and $\psi$ such that $\varphi \in L_{1}$ but $\varphi \notin L_{2}$ and $\psi \in L_{2}$ but $\psi \notin L_{1}$. We may assume that $\varphi$ is written in variables $p_{i}, i=1, \ldots n$ whereas $\psi$ in $q_{i}, i=1, \ldots m$. Since $\varphi \notin L_{2}$ then $\varphi \notin L_{0}$ and $\psi \notin L_{1}$ then $\psi \notin L_{0}$. 
Obviously $\varphi \vee \psi \in L_{1}$ and $\varphi \vee \psi \in L_{2}$. Then $\varphi \vee \psi \in L_{0}$.

From the above theorems, one may conclude that there is a very simple semantic way of identifying Halldén complete (and incomplete) logics. Each homogeneous frame determines a Halldén complete logic, whereas the class of two frames which are mutually irreducible determines a Halldén incomplete one.

Below, we characterize special families of finite, homogeneous frames for the well-known modal logics. Our interest is limited to finite and connected frames only.

Definition 7. A frame $\mathfrak{F}:=\langle W, R\rangle$ is connected if for any $x, y \in W$ there are $x_{1}, x_{2}, \ldots, x_{n} \in W$ such that $x R x_{1}$ and $x_{1} R x_{2}$ and $\ldots$ and $x_{n} R y$.

We also define set $R(x)$ of neighboring points for $x \in W$ :

$$
R(x)=\{y \in W: x R y\} .
$$

Lemma 4. Let $\mathfrak{F}:=\langle W, R\rangle$ be a finite, connected and homogeneous frame. Then

1. for any $x, y \in W, \operatorname{card} R(x)=\operatorname{card} R(y)$,

2. if exists an $x \in W$ such that $x R x$, then $y R y$ for all $y \in W$.

Proof: Since $\mathfrak{F}:=\langle W, R\rangle$ is a homogeneous frame, then for every automorphism $f$ if $x R y$ then $f(x) R f(y)$. So, $\operatorname{card} R(x)=\operatorname{card} R(f(x))$. If $x R x$ for some $x \in W$ then $f(x) R f(x)$. It means that if there is a reflexive point in a frame, then all points must be reflexive.

Very small homogeneous frames $\mathfrak{F}:=\langle W, R\rangle$ are simple to describe. Let $W=\{x\}$. Then we have two possibilities: $x R x$ or $\neg x R x$. A reflexive point is denoted by $\circ$, an irreflexive one by $\bullet$. Then we get $L(\circ)=$ Triv and $L(\bullet)=V e r$. Obviously these two logics are Halldén complete.

Let $W=\{x, y\}, x \neq y$. Similarly as before there are possible only two cases:

1. $R$ is symmetric and reflexive - then we have the frame $\circ-\circ$,

2. $R$ is symmetric and irreflexive

The situation that $x R y$ and $\neg y R x$ is not allowed, because then the frame would not be homogeneous. 
Let $W=\left\{x_{1}, x_{2}, x_{3}\right\}, x_{i} \neq x_{j}$ for $i \neq j, i, j=1,2,3$. Suppose $x_{1} R x_{2}$ and $x_{2} R x_{3}$ but $\neg x_{3} R x_{1}$. Then the frame is not homogeneous since we cannot map the point $x_{1}$ onto $x_{2}$, Indeed, if $f\left(x_{1}\right)=x_{2}$ then also $f\left(x_{2}\right)=x_{3}$. Function $f$ is onto, then it must hold $f\left(x_{3}\right)=x_{1}$. But then we get $x_{2} R x_{3}$ and $\neg f\left(x_{2}\right) R f\left(x_{3}\right)$. The simple example shows that finite homogeneous frames do not have last points, so they are, somehow, circular. Formally, we define:

Definition 8. A circular frame $\mathfrak{C}_{n}^{K}:=\left\langle W_{n}, R_{n}\right\rangle, n \geq 3$ is defined as follows:

$$
\begin{aligned}
& W_{n}:=\left\{x_{1}, x_{2}, \ldots, x_{n}\right\}, \\
& R_{n} \supseteq\left\{\left(x_{i}, x_{i+1}\right), i=1,2, \ldots, n-1\right\} \cup\left\{\left(x_{n}, x_{1}\right)\right\} .
\end{aligned}
$$

Additionally we define:

- A circular reflexive and/or symmetric frame is the circular frame whose relation $R_{n}$ is also reflexive and/or symmetric. Such frames are denoted as $\mathfrak{C}_{n}^{K T B}, \mathfrak{C}_{n}^{K T}$ or $\mathfrak{C}_{n}^{K B}$, respectively.

Below, in Fig. 1 diagrams of four distinct circular frames $\mathfrak{C}_{n}$ are presented. One may notice that

OBSERVATION 1. Each circular frame is homogeneous.

\section{Halldén completeness in $N E X T(\mathrm{KTB})$}

In this section we study homogeneous reflexive and symmetric Kripke frames. We shall prove a stronger version of Lemma 3 for homogeneous $K T B$-frames. We shall take advantage of characteristic formulas. Let us notice that in a connected $K T B$-frame each point $x$ may be treated as a root. It means that any other point $y$ is $R^{n}$ accessible from $x$ for some $n \geq 0$. Moreover, in finite, connected and homogeneous $K T B$-frame $\mathfrak{F}$, the formula $\kappa_{x}$ is satisfiable at any $y \in W$.

For inhomogeneous frames, however, we get:

Lemma 5. Let $\mathfrak{F}:=\langle W, R\rangle$ be a KTB-Kripke frame, which is finite, connected and inhomogeneous. Suppose that card $R(x) \neq \operatorname{card} R(y)$ for some $x, y \in W$. Then the formula $\kappa_{x}$ is not satisfiable at $y$. 

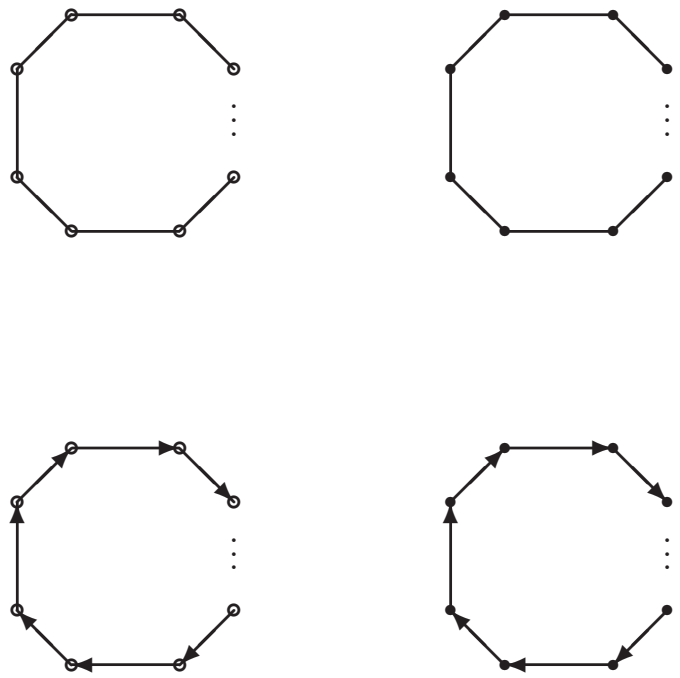

Fig. 1. The diagrams of different circular frames with $n$ points.

Proof: Let $\mathfrak{F}:=\langle W, R\rangle$ be a $K T B$ - frame, which is finite, connected and inhomogeneous. Then there exists two points $x, y \in W$ such that for any function $f: W \rightarrow W$ being a bijection such that $f(x)=y, f$ is not a homomorphism. Hence, for example, there exists a point $x_{1} \in W$ such that $x R x_{1}$ but $\neg y R f\left(x_{1}\right)$. Then the formula $\delta_{\mathfrak{F}}$ contains the subformulas $p_{x} \rightarrow \diamond p_{x_{1}}$ and $p_{y} \rightarrow \neg \vee p_{f\left(x_{1}\right)}$. If we want the formula $\kappa_{x}$ to be satisfiable at $y$ then we have to accept $y \models p_{x}$. We want to follow the previous valuation (taken from the characteristic formula), but then we have a problem. Since $\neg y R f\left(x_{1}\right)$ then the sub-formula $p_{x} \rightarrow \diamond p_{x_{1}}$ will be false at $y$ and the whole formula $\kappa_{x}$ will be falsified at $y$ as well. The same situation will hold for any other valuation. We conclude that $\kappa_{x}$ is not satisfiable at the point $y$. A similar proof will be provided if $f(x)=y$ and $\neg x R x_{1}$ but $y R f\left(x_{1}\right)$. 
Theorem 2. Let $\mathfrak{F}:=\langle W, R\rangle$ be KTB-Kripke frame, which is finite and connected. Logic $L(\mathfrak{F})$ is Halldén complete iff the frame $\mathfrak{F}$ is homogeneous.

Proof. The reverse implication is proven in the proof of Lemma 3. Then we have to prove the simple implication, only. Suppose that the frame $\mathfrak{F}$ is not homogeneous. Let $\operatorname{card} W=n$ and let $x, y \in W$ be such points that for any bijection $f: W \rightarrow W$ such that $f(x)=y$, it is not a homomorphism. Let us consider the negation of $\kappa_{x}$ and $\kappa_{y}$ appropriately.

$$
\varphi(p):=\square^{n} \delta_{\mathfrak{F}} \rightarrow \neg p_{x} \quad \text { and } \quad \psi(q):=\square^{n} \delta_{\mathfrak{F}} \rightarrow \neg q_{y}
$$

Writing $\varphi(p)$ we mean that the formula $\varphi$ is written in variables $p$ 's, appropriately $\psi$ in $q$ 's. From (1.1) we get:

$$
\begin{aligned}
& x \not \models \varphi(p), \\
& y \not \models \psi(q) .
\end{aligned}
$$

Then $\varphi(p) \notin L(\mathfrak{F})$ and $\psi(p) \notin L(\mathfrak{F})$.

From Lemma 5 we conclude that

$$
y \models \varphi(p), \quad \text { and } \quad x \models \psi(q) .
$$

Hence

$$
x \models \varphi(p) \vee \psi(q) \quad \text { and } \quad y \models \varphi(p) \vee \psi(q) .
$$

Let $z \in W, z \neq x$ and $z \neq y$. If there is an automorphism $g$ such that $g(x)=z$ then there is a valuation such that $z \not \models \varphi(p)$. But then $z \models \psi(q)$ (it follows from the existence of $g$ that there is no automorphism mapping $y$ to $z$ ). Hence

$$
z \models \varphi(p) \vee \psi(q) .
$$

Similarly we prove (3.5) if there is an automorphism $h$ mapping $y$ to $z$. Now, suppose that there is no automorphism mapping $x$ to $z$ nor $y$ to $z$. Then at $z$ both the formulas $\varphi(p)$ and $\psi(q)$ are true for any valuation. Hence again (3.5) is true.

From (3.4)-(3.5) we get that the disjunction $\varphi(p) \vee \psi(q)$ is valid in $\mathfrak{F}$. Then $\varphi(p) \vee \psi(q) \in L(\mathfrak{F})$ what involves the Halldén incompleteness of the logic $L(\mathfrak{F})$.

If a tabular logic is determined by a finite class of finite and mutually irreducible $K T B$-frames (having more than one frame) then by Theorem 1 it is not Halldén complete. From Theorem 2 we conclude: 
Corollary 1. A Kripke complete and tabular logic from NEXT(KTB), which is Halldén complete must be determined by one homogeneous Kripke frame.

\subsection{Logics determined by circular reflexive and symmetric frames}

Initially we confine ourselves to the circular reflexive and symmetric frames $\mathfrak{C}_{n}^{K T B}, n \geq 3$. The class of possible p-morphisms between such frames is easy to describe.

LemMA 6. Let $\mathfrak{C}_{k n}^{K T B}:=\left\langle W_{k n}, R\right\rangle, W_{k n}:=\left\{x_{1}, \ldots, x_{k n}\right\}, k \geq 2$ and $\mathfrak{C}_{n}^{K T B}:=\left\langle W_{n}, R^{\prime}\right\rangle, W_{n}:=\left\{x_{1}^{\prime}, \ldots, x_{n}^{\prime}\right\}$ be two circular frames. Then the following function:

$$
f\left(x_{j}\right)=x_{i}^{\prime} \quad \text { iff } \quad i=j[\bmod (n)] ; \text { for any } \quad i \leq n, j \leq k n
$$

is a p-morphism from $\mathfrak{C}_{k n}^{K T B}$ to $\mathfrak{C}_{n}^{K T B}$.

Proof. Function $f$ is onto. Let $x_{j} R x_{j+1}$. Obviously, $f\left(x_{j}\right) R^{\prime} f\left(x_{j+1}\right)$ since $f\left(x_{j}\right)=x_{j[\bmod (n)]}^{\prime}, f\left(x_{j+1}\right)=x_{j+1[\bmod (n)]}^{\prime}$ and $x_{j[\bmod (n)]}^{\prime} R^{\prime} x_{j+1[\bmod (n)]}^{\prime}$. Now, we check the condition (p3) of p-morphism. Let $f\left(x_{j}\right) R^{\prime} x_{i}$. If $f\left(x_{j}\right)=$ $x_{i}$ then the thesis is trivial. Let $f\left(x_{j}\right) \neq x_{i}$ and suppose $f\left(x_{j}\right)=x_{i+1}$ (it could be also $f\left(x_{j}\right)=x_{i-1}$, but it is analogous). Then we take the point $x_{j-1}$ if $j>2$ (or $x_{k n}$ if $j=1$ ). We get $x_{j} R x_{j-1}$ and $f\left(x_{j-1}\right)=x_{i}^{\prime}$ (or $x_{1} R x_{k n}$ and $f\left(x_{k n}\right)=x_{1}^{\prime}$, appropriately).

On the other hand it holds.

LEMma 7. Let $m>n \geq 3$ and suppose that there is a p-morphism from $\mathfrak{C}_{m}^{K T B}$ to $\mathfrak{C}_{n}^{K T B}$. Then $m$ is divisible by $n$.

Proof. It is similar to the proof of Lemma 20 from [13] or Thm. 6.4 from [7] for wheel frames. The clue of the proof is that it is impossible to glue two neighboring points and map them onto a point from the smaller circle, since then the condition (p3) of p-morphism does not hold.

Before we take advantage of Lemma 2 we shall prove:

Lemma 8. Let $\mathfrak{C}_{n}^{K T B}:=\langle W, R\rangle, n \geq 3$ be given. Then its dual $\left(\mathfrak{C}_{n}^{K T B}\right)^{*}:=$ $\left\langle 2^{W}, \cap, \cup,-, I, \emptyset, W\right\rangle$ is simple. 
Proof. We show that for any proper subset $X \subset W, X \neq I(X)$. That will mean that there are no open elements (and filters) in $\left(\mathfrak{C}_{n}^{K T B}\right)^{*}$. Let us remind the definition of the interior operation:

$$
I(X)=\left\{x \in W: \forall_{y}(x R y \Rightarrow y \in X)\right\} .
$$

If $X:=\left\{x_{1}\right\}$ then $I(X)=\emptyset$. If $X:=\left\{x_{1}, x_{2}\right\}$ then again $I(X)=\emptyset$ because each of them sees other point. Let $X:=\left\{x_{1}, x_{2}, x_{3}\right\}$ and $x_{2}$ sees itself, $x_{1}$ and $x_{3}$ and no other point. Let card $W>3$ and suppose that $x_{3} R x_{4}$. Then $x_{3} \notin I(X)$. Hence $I(X) \neq X$. If $\operatorname{card} W=3$ then $I(X)=X$, but then the filter generated by $X$ is not proper. The analogous situation holds for larger sets $X \subset W$. Eventually, only in two cases we get $X=I(X)$ for $X:=\emptyset$ or $X:=W$.

One may conclude from Lemmas 8 and 2 that

Corollary 2. For any prime numbers $i \neq j$ such that $i, j \geq 3$ we get $L\left(\mathfrak{C}_{i}^{K T B}\right) \nsubseteq L\left(\mathfrak{C}_{j}^{K T B}\right)$ and $L\left(\mathfrak{C}_{j}^{K T B}\right) \nsubseteq L\left(\mathfrak{C}_{i}^{K T B}\right)$.

Further, from Lemma 3 one may easily conclude:

COROllary 3. Each logic determined by one circular (reflexive and symmetric) Kripke frame is Halldén complete. Moreover, logics determined by one circular (reflexive and symmetric) Kripke frame having prime number of points form an anti-chain.

One may notice that the formula $\left(a_{l} t_{3}\right)$ is valid in any circular reflexive and symmetric Kripke frame. Then, if fact, we may conclude:

COROLlary 4. There are countably many Halldén complete logics in $N E X T\left(\right.$ KTB.alt $\left._{3}\right)$.

Moreover we obtain:

Corollary 5. All tabular and Halldén complete logics in $N E X T\left(\mathbf{K T B} \mathbf{a l t}_{\mathbf{3}}\right)$ are determined by the circular frames: $\left\{\mathfrak{C}_{n}^{K T B}, n \geq 3\right\}$ or by the frames: $\circ--\circ$ or 0 .

Two logics, determined by different circular frames with prime number of points, are mutually incomparable. Thus, their intersection determines logic which is Halldén incomplete (see Theorem 1). Then we get: 
Corollary 6. There are countably many Halldén-incomplete logics in $N E X T\left(\right.$ KTB.alt $\left._{3}\right)$.

REMARK 1. Since we have defined an anti-chain of Halldén complete logics in NEXT(KTB.alt $\left.\mathbf{H}_{3}\right)$ then it might seem to be possible to define a continuum of logics by taking their infinite intersections. But the whole family $N E X T$ (KTB.alt $\mathbf{B}_{\mathbf{3}}$ ) is countably infinite only, hence as well the family of Halldén incomplete logics, see [2].

\subsection{Halldén completeness in $N E X T\left(K_{T B}\right.$.alt $\left.\mathbf{n}\right)$ for $n \geq 4$}

Among homogeneous Kripke frames one may distinguish other than the circular ones. There are plenty of examples of such frames. Let us still consider reflexive and symmetric circular frames. After some modification of their definition, we may obtain homogeneous frames with established degree of branching.

Since the axioms $\left(a l t_{n}\right)$ are valid in the frames whose points see at most $n$ other points (including itself) then we have the following inclusion:

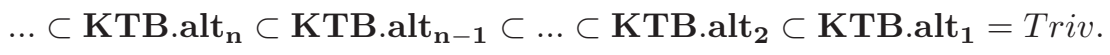

Below, we modify the reflexive and symmetric circular Kripke frames as follows:

Definition 9. A double circular reflexive and symmetric frame $\mathfrak{D C}_{2 n}^{K T B}:=$ $\left\langle W_{2 n}, R_{2 n}\right\rangle, n \geq 3$ is defined as follows:

$$
\begin{aligned}
W_{2 n}:= & \left\{x_{1}, x_{2}, \ldots, x_{n}\right\} \cup\left\{x_{1}^{\prime}, x_{2}^{\prime}, \ldots, x_{n}^{\prime}\right\}, \\
R_{2 n}:= & \left\{\left(x_{i}, x_{j}\right):|i-j| \leq 1, i, j=1, \ldots, n\right\} \cup\left\{\left(x_{1}, x_{n}\right)\right\} \cup\left\{\left(x_{n}, x_{1}\right)\right\} \cup \\
& \left\{\left(x_{i}^{\prime}, x_{j}^{\prime}\right):|i-j| \leq 1, i, j=1, \ldots, n\right\} \cup\left\{\left(x_{1}^{\prime}, x_{n}^{\prime}\right)\right\} \cup\left\{\left(x_{n}^{\prime}, x_{1}^{\prime}\right)\right\} \cup \\
& \left\{\left(x_{i}, x_{i}^{\prime}\right): i=1, \ldots, n\right\} \cup\left\{\left(x_{i}^{\prime}, x_{i}\right): i=1, \ldots, n\right\} .
\end{aligned}
$$

As an example the diagram of $\mathfrak{D C}_{16}^{K T B}$ is presented in Fig. 2.

The logics determined by frames $\mathfrak{D C}_{2 n}^{K T B}, n \geq 3$ belong to the family $N E X T\left(\mathbf{K T B}_{\mathbf{a}} \mathbf{a l t}_{\mathbf{4}}\right)$, which contains the family $N E X T\left(\mathbf{K T B}_{\mathbf{a}} \mathbf{a l t}_{\mathbf{3}}\right)$. For them we may repeat the reasoning from Section 3.1. The class of p-morphisms are similar to the one described in Lemmas 6 and 7 . We 


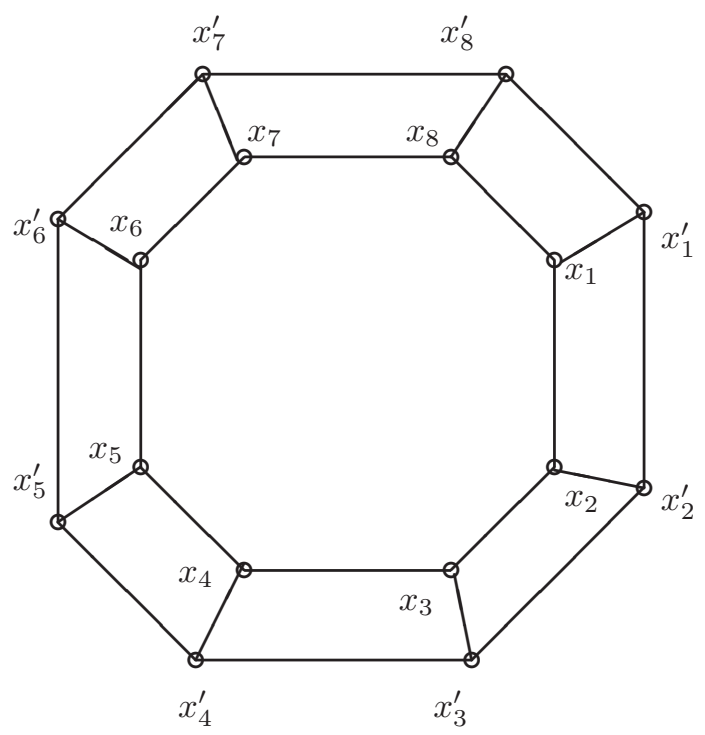

Fig. 2. The diagram of reflexive, symmetric double circular frame $\mathfrak{D C}_{16}^{K T B}$

may also prove that the dual algebra $\left(\mathfrak{D C} \mathfrak{C}_{2 n}^{K T B}\right)^{*}$ for $n \geq 3$ is simple, what involves that the homogeneous frames $\mathfrak{D C}_{2 n}^{K T B}$ with $n$-prime, determine logics that form an anti-chain. Then we get:

Corollary 7. There are countably many Halldén complete logics in $N E X T\left(\right.$ KTB.alt $\left._{4}\right) \backslash N E X T\left(\right.$ KTB.alt $\left._{3}\right)$.

By Lemmon theorem we also obtain:

COROLlary 8. There are countably many Halldén-incomplete logics in $N E X T\left(\right.$ KTB.alt $\left._{\mathbf{4}}\right) \backslash N E X T\left(\right.$ KTB.alt $\left._{\mathbf{3}}\right)$.

REMARK 2. The cardinality of the whole family NEXT(KTB.alt 4 ) is that of the continuum (see [9]). We have defined an anti-chain of Halldén complete logics in NEXT(KTB.alt $\left.{ }_{4}\right)$ and it seems to be possible to define a continuum of logics by taking their infinite intersections. However each double circular frame $\mathfrak{D C}_{2 n}^{K T B}$ is reducible to the circular one $\mathfrak{C}_{n}^{K T B}$ (the suitable $p$-morphism glues the points $x_{i}$ and $x_{i}^{\prime}$ for $\left.i=1,2, \ldots, n\right)$. Then 
we conclude again that the class of logics determined by double circular frames is countably infinite only. Hence the class of Halldén incomplete logics defined by us is also countably infinite.

The idea presented above of constructing of double circular frames from circular ones may be generalized. Actually, the double circular frame is isomorphic to a direct product of circular frame and two element cluster. We recall the definition.

DeFinition 10. The direct product of frames $\mathfrak{F}_{1}:=\left\langle W_{1}, R_{1}\right\rangle$ and $\mathfrak{F}_{2}:=$ $\left\langle W_{2}, R_{2}\right\rangle$ is the frame $\mathfrak{F}_{1} \times \mathfrak{F}_{2}:=\left\langle W_{1} \times W_{2}, R_{1} \otimes R_{2}\right\rangle$, where symbol ' $\times$ ' denotes Cartesian product, and

$$
\left(x_{1}, x_{2}\right) R_{1} \otimes R_{2}\left(y_{1}, y_{2}\right) \text { iff } x_{1} R_{1} y_{1} \text { and } x_{2} R_{2} y_{2},
$$

for any $x_{1}, y_{1} \in W_{1}$ and $x_{2}, y_{2} \in W_{2}$.

An $n$-element Kripke frame $\mathfrak{F}:=\langle W, R\rangle$ is an $n$-element cluster if for any $x, y \in W$ we have $x R y$. Such an $n$-element cluster is denoted $\mathfrak{C l}_{n}$. Of course, each cluster is also an homogeneous Kripke frame. From the above definition we notice that $\mathfrak{D C}_{n}^{K T B} \cong \mathfrak{C}_{n}^{K T B} \times \mathfrak{C l}_{2}$. Following the above idea we will consider the following direct products:

$$
\mathfrak{C}_{n}^{K T B} \times \mathfrak{C l}_{k}, n \geq 3 \text { and } k \in \mathbb{N} .
$$

OBSERVATION 2. Each direct product $\mathfrak{C}_{n}^{K T B} \times \mathfrak{C l}_{k}$ is a homogeneous frame and each point of this frame sees exactly $k+2$ points (including itself).

The logics determined by the frames $\mathfrak{C}_{n}^{K T B} \times \mathfrak{C l}_{k}, n \geq 3 k \in \mathbb{N}$ belong to the family $N E X T($ KTB.alt $\mathbf{k + 2})$. We generalize Corollaries 7 and 8 as follows:

Corollary 9. There are countably many Halldén complete logics in $N E X T\left(\mathbf{K T B}_{\mathbf{a l t}} \mathbf{m}\right) \backslash N E X T\left(\mathbf{K T B}_{\mathbf{a}} \mathbf{a l t}_{\mathbf{m}-\mathbf{1}}\right)$ for any $m \geq 4$.

Corollary 10. There are countably many Halldén-incomplete logics in $N E X T\left(\right.$ KTB.alt $\left._{\mathbf{m}}\right) \backslash N E X T\left(\mathbf{K T B}_{\mathbf{a l t}} \mathbf{m - 1}\right)$ for any $m \geq 4$.

\subsection{Halldén completeness in $N E X T\left(K T B .4_{\mathbf{n}}\right)$}

The Brouwer logic is called a non-transitive logic since it is determined by the class of reflexive and symmetric frames, which are allowed to be 
non-transitive. But also its $n$-transitive normal extensions are studied. Obviously, KTB. $\mathbf{4}_{\mathbf{1}}=\mathbf{S} 5$ and the following inclusions hold:

$$
\mathrm{KTB} \subset \ldots \subset \mathrm{KTB} .4_{\mathrm{n}+1} \subset \mathrm{KTB}_{4_{\mathrm{n}}} \subset \ldots \subset \mathrm{KTB} .4_{2} \subset \mathrm{KTB} .4_{1} .
$$

The logic S5 is determined by Kripke frames which are clusters. For any $n>1$ the cluster $\mathfrak{C l}_{n}$ is reducible to $\mathfrak{C l}_{n-1}$. Each cluster is a homogeneous frame and determines a normal extension of S5. Then it is quite clear that S5 has countably many normal extensions all of which are Halldén complete (see Introduction). Then we may study weaker logics. We start with the logic KTB.4.

We modify reflexive and symmetric circular frames by adding connections between some points. The new frame we call a circular-asteroid frame.

Definition 11. A circular-asteroid reflexive and symmetric frame $\mathfrak{C A}_{n}:=$ $\left\langle W_{n}, R_{n}\right\rangle, n \geq 5$ is defined as follows:

$$
\begin{aligned}
W_{n}:= & \left\{x_{1}, x_{2}, \ldots, x_{n}\right\}, \\
R_{n}:= & \left\{\left(x_{i}, x_{j}\right):|i-j| \leq 1, i, j=1,2, \ldots, n\right\} \cup\left\{\left(x_{1}, x_{n}\right)\right\} \cup\left\{\left(x_{n}, x_{1}\right)\right\} \cup \\
& \left\{\left(x_{i}, x_{j}\right): 3 \leq|i-j| \leq n-3, i, j=1,2, \ldots, n\right\} .
\end{aligned}
$$

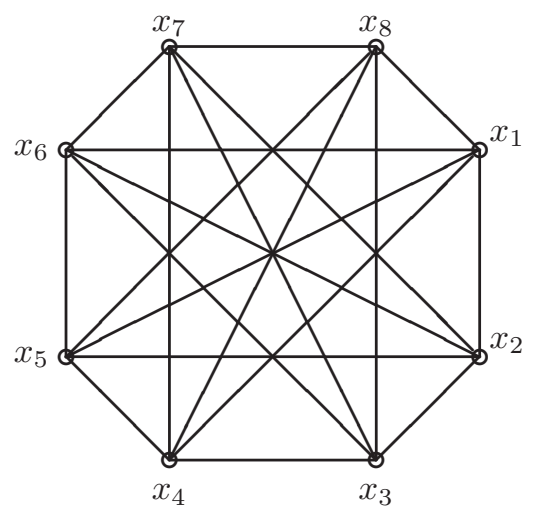

Fig. 3. The diagram of reflexive, symmetric circular-asteroid frame $\mathfrak{C A} \mathfrak{R}_{8}^{K T B}$ 
Each point $x_{i}, i \leq n$ of such a frame sees almost all other points with the exception of $x_{i+2[\bmod n]}$ and symmetrically $x_{n+i-2[\bmod n]}$. Then reflexive, symmetric circular-asteroid frame is not a cluster, but it is $K T B 4_{2}$-frame. Obviously, circular-asteroid, reflexive and symmetric frames are homogeneous. Although in these frames there are more connections than in the circular ones, surprisingly the class of possible p-morphisms is the same. First, let us notice that the analogues of Lemmas 6 and 7 hold. Also, the dual algebra $\left(\mathfrak{C A}_{n}^{K T B}\right)^{*}$ is simple. Then it is possible to construct an anti-chain of logics in $N E X T\left(\mathbf{K T B} . \mathbf{4}_{\mathbf{2}}\right)$ which are Halldén complete. The logics, as before, will be determined by suitable frames with prime numbers of points. Then we get:

COROllary 11. There are countably many Halldén complete logics in $N E X T\left(\mathbf{K T B} .4_{2}\right) \backslash N E X T(\mathbf{S 5})$.

We may compare the above result with the negative one from [8]. The result from [8] was obtained by using wheel-frames. Our positive result from Corollary 11 is a partial solution of Problem 1 from this paper.

The class of the Halldén incomplete logics described in [8] has the cardinality of the continuum. Similarly as for wheel-frames (see [13]), we may prove that

Lemma 9. The class of logics determined by circular-asteroid, reflexive and symmetric frames has cardinality continuum.

Proof: The logics $L\left(\mathfrak{C A}_{n}^{K T B}\right)$ and $L\left(\mathfrak{C A}_{m}^{K T B}\right), m, n \geq 5$ - prime and $m \neq$ $n$ are incomparable. Let $A$ and $B$ be distinct sets of prime numbers. We show that

$$
L\left(\left\{\mathfrak{C A}_{n}^{K T B}, n \in A\right\}\right) \neq L\left(\left\{\mathfrak{C A}_{n}^{K T B}, n \in B\right\}\right) .
$$

Let $n_{1} \in A$ and $n_{1} \notin B$. By Rautenberg's theorem we know that each finite frame splits the lattice $N E X T\left(\mathbf{K T B} .4_{2}\right)$ (see [14]). The splitting pair is the following $\left(L\left(\mathfrak{C A}_{n}^{K T B}\right), \mathbf{K T B} .4 .2 \oplus \neg \kappa_{\mathfrak{C A}} \mathfrak{A}_{n}^{K T B}\right)$. The negation of the characteristic formula $\kappa_{\mathfrak{C A}^{K} \mathfrak{A}_{1}}^{K T B}$ it valid in all frames $\mathfrak{C A}_{n}^{K T B}, n \in B$, but is not in $\mathfrak{C A}_{n_{1}}^{K T B}$. Then we may distinguish the two logics $L\left(\left\{\mathfrak{C A}_{n}^{K T B}, n \in\right.\right.$ $A\})$ and $L\left(\left\{\mathfrak{C A}_{n}^{K T B}, n \in B\right\}\right)$ from each other. 
Then we get a similar result to the one from [8]:

COROLlary 12. There are uncountably many Halldén-incomplete logics in $N E X T\left(\mathbf{K T B} . \mathbf{4}_{\mathbf{2}}\right) \backslash N E X T(\mathbf{S 5})$, which are determined by circular-asteroid, reflexive and symmetric frames.

REMARK 3. The circular-asteroid frames may be modified by removing the connections between $x_{i}$ and $x_{i+3}$ and symmetrically $x_{i}$ and $x_{n+i-3}$. Then we get homogeneous frames with the largest diameter 3 . Such frames indexed with prime numbers will determine as before an anti-chain of logics in $N E X T\left(\mathbf{K T B} . \mathbf{4}_{\mathbf{3}}\right) \backslash N E X T\left(\mathbf{K T B} . \mathbf{4}_{\mathbf{2}}\right)$. Analogously, we define circularasteroid frames with a larger diameter for any $n \geq 4$.

\section{Logics determined by other circular Kripke frames}

In this section we briefly describe Halldén complete logics determined by the class of circular Kripke frames which are irreflexive and antisymmetric $\left\{\mathfrak{C}_{n}^{K}, n \geq 3\right\}$, or irreflexive and symmetric $\left\{\mathfrak{C}_{n}^{K B}, n \geq 3\right\}$, or reflexive and antisymmetric $\left\{\mathfrak{C}_{n}^{K T}, n \geq 3\right\}$. The classes of existing p-morphisms for each kind of circular frames are very similar to the one from previous section. Analogous lemmas to 6,7 and 8 hold. Also one may conclude similarly to Corollary 4 that

Corollary 13. There are countably many Halldén complete logics in the families:

- $N E X T(\mathbf{K}) \backslash(N E X T(\mathbf{K T}) \cup N E X T(\mathbf{K B}))$

- $N E X T(\mathbf{K B}) \backslash N E X T(\mathbf{K T B})$

- $N E X T(\mathbf{K T}) \backslash N E X T(\mathbf{K T B})$

Similarly as in Corollary 6 we also obtain:

Corollary 14. There are countably many Halldén-incomplete logics in the families:

- $N E X T(\mathbf{K}) \backslash(N E X T(\mathbf{K T}) \cup N E X T(\mathbf{K B}))$

- $N E X T(\mathbf{K T}) \backslash N E X T(\mathbf{K T B})$

- $N E X T(\mathbf{K B}) \backslash N E X T(\mathbf{K T B})$ 


\section{Problems and questions}

In paper [1] its authors give several theorems about Halldén completeness of modal logics which are determined by special Kripke frames. All the theorems are, however, only implications and the authors regret this fact. Hence our Theorem 2 for $K T B$-frames is important in this area of research. Since it was possible to get a theorem in a shape of equivalence for some class of some special frames (not only homogeneous, but also reflexive and symmetric) we propose to study less general classes of Kripke frames than in the mentioned paper. For example, one may study classes of frames closed under p-morphic fusions (but only transitive), or classes closed under direct products. Strong theorems in the form of equivalence characterizing Halldén-completeness will be very useful for studying logics with interpolation. We briefly recall here that the logics which have only one Post complete extension and are not Halldén-complete do not have interpolation as Schumm [15] showed. Although this result concerns non-normal extensions of modal logics, we think that it may be helpful also in the case of normal ones. Hence our Theorem 2 is a starting point for recognizing logics with interpolation in $N E X T(\mathbf{K T B})$.

Further, let us notice, that our method of defining Halldén-complete normal modal logics serves to yield only $\aleph_{0}$ such logics. Hence the following question seems to be natural:

QUESTION 1. Is it possible to construct an uncountable family of Halldén complete logics in $N E X T\left(\mathbf{K T B} . \mathbf{a l t}_{\mathbf{n}}\right)$ for $n \geq 4$ or in $N E X T\left(\mathbf{K T B} . \mathbf{4}_{\mathbf{n}}\right)$, $n \geq 2$ or in $N E X T(\mathbf{K}) \backslash(N E X T(\mathbf{K T}) \cup N E X T(\mathbf{K B}))$ or in $N E X T(\mathbf{K T}) \backslash$ $N E X T(\mathbf{K T B})$ or in $N E X T(\mathbf{K B}) \backslash N E X T(\mathbf{K T B})$ or in $N E X T(\mathbf{K T B})$ ?

A positive answer to the question is connected with defining the so-called conservative formulas, see [4], p.468. In our opinion, in the case of logic KTB it is very difficult to find such formulas.

ACKnowledgment. The author would like to thank Yutaka Miyazaki for fruitful discussion on Halldén completeness of modal logics. 


\section{References}

[1] J. F. A. K. van Benthem, I. I. Humberstone, Halldén-completeness by Gluing of Kripke Frames, Notre Dame Journal of Formal Logic 24, No 4 (1983), pp. 426-430.

[2] M. Byrd, D. Ullrich, The extensions of BAlt 3 , Journal of Philosophical Logic 6 (1977), pp. 109-117.

[3] A. Chagrov, M. Zakharyaschev, On Halldén-completeness of intermediate and modal logics, Bulletin of the Section of Logic 19:1(1990), pp. 21-23.

[4] A. Chagrov, M. Zakharyaschev, Modal Logic, Oxford Logic Guides 35 (1997).

[5] S. Halldén, On the semantic non-completeness of certain Lewis calculi, The Journal of Symbolic Logic 16 (1951), pp. 127-129.

[6] V. A. Jankov, Conjunctively indecomposable formulas in propositional calculi, Izv, Akad. Nauk USSR Ser. Mat. 33 (1969), pp. 18-38.

[7] Z. Kostrzycka, On Modal Systems in the Neighbourhood of the Brouwer Logic, Acta Universitatis Wratislaviensis No 3238, Logika 25, Wydawnictwo Uniwersytetu Wrocawskiego, Wrocaw (2010).

[8] Z. Kostrzycka, On interpolation and Halldén-completeness in NEXT(KTB), Bulletin of the Section of Logic 41:1/2 (2012), pp. 23-32.

[9] Z. Kostrzycka, Y. Miyazaki, Normal modal logics determined by aligned clusters, submitted.

[10] S. A. Kripke, Semantical analysis of modal logic I., Zeitschr. f. math. Logik und Grundlagen d. Math. 9 (1963), pp. 67-96.

[11] E. J. Lemmon, A note on Halldén-incompleteness, Notre Dame Journal of Formal Logic, VII, No 4, (1966), pp. 296-300.

[12] J. C. C. McKinsey, Systems of modal logics which are not unreasonable in the sense of Hallden, Journal of Symbolic Logic 18 (1953), pp. 109-113.

[13] Y. Miyazaki, Normal modal logics containing KTB with some finiteness conditions, Advances in Modal Logic 5 (2005), pp. 171-190.

[14] W. Rautenberg, Splitting lattices of logics, Archiv für Mathematische logik 20 (1980), pp. 155-159.

[15] G. F. Schumm, Some failures of interpolatin in modal logic, Notre Dame Journal of Formal Logic 27/1 (1986), pp. 108-110.

University of Technology

Luboszycka 3, 45-036, Opole, Poland

e-mail: z.kostrzycka@po.opole.pl 